
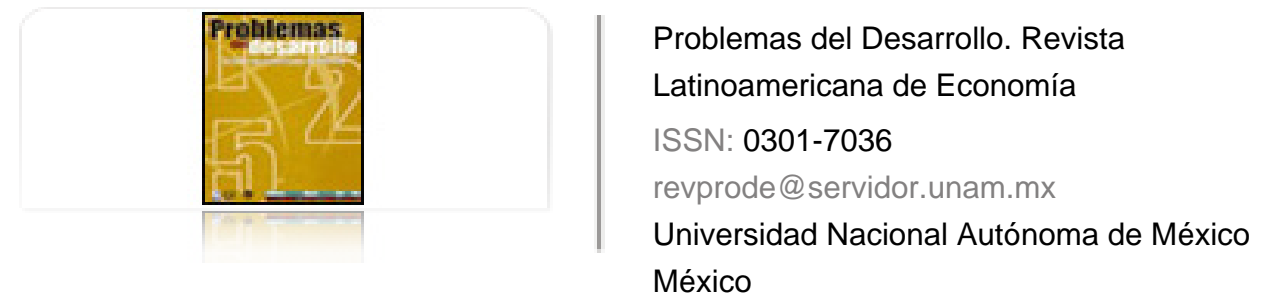

Absell, Christopher David

El alba-tcp mirado con buenos ojos

Problemas del Desarrollo. Revista Latinoamericana de Economía, vol. 43, núm. 169, abril-junio, 2012, pp. 73-95

Universidad Nacional Autónoma de México

Distrito Federal, México

Disponible en: http://www.redalyc.org/articulo.oa?id=11823064004

- Cómo citar el artículo

- Número completo

- Más información del artículo

Página de la revista en redalyc.org

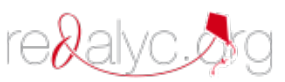

Sistema de Información Científica

Red de Revistas Científicas de América Latina, el Caribe, España y Portugal Proyecto académico sin fines de lucro, desarrollado bajo la iniciativa de acceso abierto 
Revista Problemas del Desarrollo, 169 (43), abril-junio 2012

\title{
EL ALBA-TCP MIRADO CON BUENOS OJOS ${ }^{\mathrm{I}}$
}

\author{
Christopher David Absell*
}

Fecha de recepción: 14 de febrero de 2011. Fecha de aceptación: 27 de septiembre de 2011.

\section{RESUMEN}

El surgimiento de la Alianza Bolivariana para los Pueblos de Nuestra América-Tratado de Comercio de los Pueblos (ALBA-TCP) representa una novedad de importancia regional e internacional; sin embargo hasta la fecha el trabajo académico sobre la organización ha sido escaso y se ha caracterizado por tener un enfoque metodológico descriptivo carente de investigación empírica comprensiva. En este artículo sostengo que este enfoque metodológico ha imbuido la literatura de un sesgo positivo a favor del ALBA-TCP y sus proyectos de desarrollo. El objetivo de este artículo es examinar esta literatura para determinar una agenda de investigación para el estudio del ALBA-TCP.

Palabras clave: Alianza Bolivariana para los Pueblos de Nuestra América-Tratado de Comercio de los Pueblos (ALBA-TCP); América Latina; integración económica; agenda de investigación; reseña de la literatura.

\section{The AlbA-TCP LOOKING With KeEn Eyes}

\begin{abstract}
The emergence of the Bolivarian Alliance for the Peoples of our America-People's Trade Agreement (ALBA-TCP) represents an important regional and international political development, yet to date scholarly work on the subject has been scarce and characterised by a descriptive methodological approach which lacks comprehensive empirical research. Here I argue that such a methodological approach has imbued the literature with a positive bias in favour of the ALBA-TCP and its development projects. The aim of this article is to dissect this work in order to determine a research agenda for the future study of the ALBA-TCP.

Keywords: Bolivarian Alliance for the Peoples of Our America-People's Trade Agreement (ALBA-TCP); Latin America; economic integration; research agenda; literature review.

1 El autor agradece a los evaluadores anónimos por sus valiosas contribuciones, a la Dra. Heloise Weber (Universidad de Queensland) por sus comentarios y a Clara Álvarez Ríos por su ayuda con la traducción.

* Estudiante de la Maestría en Relaciones Internacionales y Cooperación UE-AL del Centro Universitario de Ciencias Económico Administrativas de la Universidad de Guadalajara. Correo electrónico: cabsell@gmail.com
\end{abstract}


Christopher David Absell

\section{L'ALBA-TCP BIEN VU \\ Résumé}

Le surgissement de l'Alliance Bolivarienne pour les peuples de notre Amérique - Traité de Commerce des Peuples (ALBA-TCP) constitue une nouveauté importante aux niveaux régional et international, cependant jusqu'à aujourd'hui, le travail académique sur cette organisation a été peu abondant et s'est caractérisé par une perspective méthodologique descriptive à laquelle fait défaut une recherche empirique compréhensive. Dans cet article, je soutiens que cette perspective méthodologique a imprégné la littérature sur le sujet d'un a priori positif à l'égard de l'ALBA-TCP et de ses projets de développement. L'objectif de cet article est d'examiner cette littérature pour déterminer un agenda de recherche pour l'étude de l'ALBA-TCP.

Mots clés : Alliance bolivarienne pour les peuples de notre Amérique - Traité de Commerce des Peuples (ALBA-TCP) ; Amérique latine ; intégration économique ; agenda de recherche ; compte rendu de la littérature

\section{A ALBA-TCP VISTA COM BONS OLHOS \\ Resumo}

O surgimento da Aliança Bolivariana para os povos da nossa América - Tratado de Comércio dos Povos (ALBA-TCP) representa uma novidade de importância regional e internacional, no entanto, até hoje o trabalho acadêmico sobre a organização foi escasso e se caracterizou por ter um enfoque metodológico descritivo carente de pesquisa empírica compreensiva. Neste artigo, sustento que este enfoque metodologioco dotou a literatura de um viés positivo a favor da ALBA-TCP e seus projetos de desenvolvimento. $\mathrm{O}$ Objetivo deste artigo é examinar esta literatura para determinar uma agenda de pesquisa para o estudo da ALBA-TCP.

Palavras-chave: Aliança Bolivariana para os povos da nossa América - Tratado de Comércio dos Povos (ALBA-TCP), América Latina, Integração econômica; Agenda de pesquisa; Resenha da literatura.

对美洲玻利瓦尔联盟人民贸易协定的审视

摘要：美洲玻利瓦尔联盟人民贸易协定（西班牙文缩写：ALBA-TCP）的 产生是实现地区和国际发展合作的重要创新。然而, 目前对其研究还不充 分, 且研究方法仅仅是描述性的, 缺乏经验实证和综合分析。本文主张所 运用的分析方法应对ALBA-TCP及其倡导的计划做出积极评价, 目标是为分 析ALBA-TCP确立一个研究议程。

关键词: 美洲玻利瓦尔联盟人民贸易协定, 拉丁美洲, 经济一体化, 研究议 程, 文献综述。 


\section{INTRODUCCIÓN}

El surgimiento del ALBA-TCP representa una novedad de importancia regional e internacional. Desde su creación en 2004 el ALBA-TCP ha cambiado la dinámica política de América Latina. Se han puesto en duda muchas de las estrategias regionales preexistentes de integración económica y cooperación, dando prioridad a la inclusión social y la equidad económica en lugar de la mera sincronización de la política económica. Además, la naturaleza compleja y contradictoria del desarrollo del ALBA-TCP nos ha obligado a repensar las teorías preexistentes de cooperación e integración.

Mientras que el ALBA-TCP y sus proyectos se han desarrollado rápidamente durante los pocos ańos de su existencia, el trabajo académico sobre la organización no ha surgido con la misma rapidez. La literatura disponible sobre el ALBA-TCP es principalmente descriptiva en su naturaleza, depende del análisis de la documentación de la fuente primaria y carece de investigación empírica. Sostengo que este enfoque metodológico ha desembocado en una perspectiva que ve el ALBA-TCP con buenos ojos; es decir, mientras que sigue siendo crítica, esta perspectiva considera el surgimiento de la organización como una novedad positiva para el desarrollo de los pueblos de América Latina, de El Caribe y del mundo. El objetivo de este artículo es examinar la literatura sobre el ALBA-TCP para elaborar una agenda de investigación que centrará la atención en las áreas más necesitadas de investigación. Mediante la ampliación del conocimiento sobre los proyectos de desarrollo del ALBA-TCP se espera que esta mirada con buenos ojos pueda ser descartada a favor de una perspectiva basada en la verificación empírica de las hipótesis preexistentes. En la primera sección proporcionaré un breve resumen de la historia del ALBA-TCP y sus características institucionales. La segunda sección tratará sobre la naturaleza de la literatura sobre el ALBA-TCP y explorará la gama de definiciones ofrecidas por los autores y las críticas principales a la organización. Como conclusión ampliaré mi argumento y sugeriré una agenda para la investigación del ALBA-TCP.

\section{UN BREVE RESUMEN DEL ALBA-TCP}

El concepto de la Alternativa Bolivariana para los Pueblos de Nuestra América (ALBA) fue articulado por primera vez en 2001 por Hugo Chávez durante la Tercera Cumbre de los Jefes de Estado y Gobierno de la Asociación de Estados de El Caribe. Durante su discurso, Chávez rechazó el modelo neoliberal que caracterizó a otros esquemas de integración en América Latina y propuso una 
alternativa, "... la idea original de muchos otros hombres y mujeres de nuestro continente... un nuevo concepto de integración que no es nada nuevo..." (ALBA-TCP, 2009a). Durante los años siguientes el concepto fue debatido por Chávez y su mentor político, Fidel Castro, ${ }^{2}$ y pronto se convirtió en una estrategia más completa cuyo objetivo era lograr, nada menos, que la consecución de la misión histórica de Simón Bolívar, José Martí y otros "libertadores" latinoamericanos: "el Sueńo Bolivariano," la emancipación del poder imperial y la consolidación de la integración regional. El primer paso hacia la institucionalización de este proyecto se dio el 14 de diciembre de 2004, cuando Venezuela y Cuba firmaron un acuerdo y emitieron una declaración conjunta anunciando la creación del ALBA. Inicialmente enmarcado como una alternativa al Área de Libre Comercio de las Américas (ALCA $)^{3}$ que, según la declaración conjunta, sólo contribuyó a “...la profundización del neoliberalismo...” y a "...los niveles de la dependencia y subordinación sin precedente...” (ALBA-TCP, 2004a) en la región, el acuerdo también sirvió como base institucional para el comercio intrarregional que se había iniciado entre los dos países en la forma de personal médico y educacional de Cuba a cambio de petróleo subsidiado de Venezuela (ALBATCP, 2004b). Este acuerdo se consolidó aún más durante 2004 y 2005 a través de la elaboración y aplicación de un "plan estratégico" que aumentó el grado de cooperación entre los dos países (ALBA-TCP, 2005).

La institucionalización del ALBA estuvo acompañada de un fenómeno complementario político que azotó la región durante la primera década del siglo Xxi: el ascenso al poder de un grupo de gobiernos de izquierda que compartieron el enfoque ideológico del ALBA. La presencia de esta coyuntura ideológica llevó a la rápida expansión de la membresía de la organización, a partir de la adhesión de Bolivia en 2006 bajo el liderazgo de Evo Morales. Tras la incorporación de Bolivia, el título de la organización se amplió para incluir el Tratado de Comercio de los Pueblos (TCP), propuesto originariamente por Morales, que además definió a la organización como una alternativa a "...los acuerdos de libre comercio que buscan incrementar el poder y el control de las trasnacionales" (ALBA-TCP, 2006). Luego vino la suscripción de Nicaragua en 2007 poco después de la elección de Daniel Ortega, cuyo primer acto oficial como presidente fue firmar un acuerdo para unirse a la organización. Se amplió su membresía a lo largo de 2008 con la inclusión de Dominica y

2 Para un buen análisis de la naturaleza de esta relación véase Azicri, 2009.

3 En inglés: The Free Trade Area of the Americas (FTAA). 
Honduras, promovido por el primer ministro Roosevelt Skerrit y el presidente Manuel Zelaya, respectivamente. Rafael Correa de Ecuador mostró interés en el proyecto antes de asumir la presidencia en 2007, pero el país siguió siendo un observador oficial hasta 2009 cuando se unió junto con San Vicente y las Granadinas y Antigua y Barbuda. En ese mismo año la organización, previamente conocida como la Alternativa Bolivariana, se convirtió en la Alianza Bolivariana (ALBA-TCP, 2009b).

Cuadro 1. Miembros del alba-tcp (Comisión Económica para América Latina y El Caribe, 2010: 23, 81)

\begin{tabular}{llrr}
\hline \multicolumn{1}{c}{ País } & \multicolumn{1}{c}{ Fecha de adhesión } & Población (2010) & PIB (2009) $^{*}$ \\
\hline República Bolivariana de Venezuela & 14 de diciembre 2004 & 29043000 & 325399,4 \\
República de Cuba & 14 de diciembre 2004 & 11203000 & 62278,6 \\
Estado Plurinacional de Bolivia & 29 de abril 2006 & 10031000 & 17340,0 \\
República de Nicaragua & 23 de febrero 2007 & 5822000 & 6149,0 \\
Commonwealth de la Dominique & 20 de enero 2008 & 67000 & 375,7 \\
República de Honduras & 25 de agosto 2008 hasta & 7621000 & 14318,2 \\
& 13 de enero 2010 & & \\
República de Ecuador & 24 de junio 2009 & 13773000 & 52021,9 \\
Antigua y Barbuda & 24 de junio 2009 & 89000 & 1097,7 \\
San Vicente y las Granadinas & 24 de junio 2009 & 109000 & 585,4 \\
\hline
\end{tabular}

* Millones de dólares estadounidenses a precio corriente de mercado.

La naturaleza institucional del ALBA-TCP se desarrolló de manera paralela a la expansión de su membresía. Alrededor del Consejo Presidencial, el cuerpo principal de toma de decisiones, surgió una estructura institucional completa: el Consejo de Ministros, que agrupa a los ministros de cada país según su área de especialización (política, social, económica); el Consejo de Movimientos Sociales, que contiene representantes de los diferentes movimientos sociales de cada país, y otros Comités que examinan áreas específicas. Estos grupos caen bajo la dirección de la Coordinación Permanente (Secretaría Ejecutiva) que coordina "las actividades de cooperación e integración" de la organización (ALBA-TCP, 2010; Romero, G., 2010). Un aspecto clave del marco institucional del ALBA-TCP es el concepto de los "proyectos grannacionales" (ALBA-TCP, 2008). Éstos están compuestos por un grupo de organizaciones pan-nacionales -"empresas grannacionales"- que trabajan en los aspectos técnicos de la integración regional como la construcción de infraestructuras, entrenamiento 
y comunicación. Un número de proyectos ya han comenzado bajo este concepto, como por ejemplo el "Proyecto Grannacional de Alfabetización y Post-Alfabetización", cuyo objeto es reducir el analfabetismo, y el "Proyecto Grannacional de Comercio Justo" que trabaja en la expansión del comercio justo y la integración económica. ${ }^{4}$

Al principio las reacciones extranjeras a la consolidación del ALBA-TCP no fueron muy pronunciadas. En 2008 un Comité del Senado de los Estados Unidos emitió un threat assessment que implícitamente se refirió al ALBA-TCP cuando observó que

“...los líderes de Bolivia, Nicaragua, y más tentativamente Ecuador, están persiguiendo agendas que destacan... el nacionalismo económico a expensas de los enfoques basados en el mercado... cada uno de estos gobiernos, en distintos grados, se ha involucrado fuertemente en la retórica anti-Estados Unidos, alineados con Venezuela y Cuba... y abogado por medidas que directamente chocan con las iniciativas de EE.uU" (Backer et al., 2009: 106).

A principios de 2010 el recién electo presidente de Honduras, Porfirio Lobo, reflejó estas preocupaciones cuando se retiró oficialmente del ALBA-TCP y señaló que "para mí sería imposible ser parte de una alianza que tiene como objetivo atacar a Estados Unidos" (El Heraldo, 2 febrero 2010)..$^{5}$ Sin embargo, estas reacciones negativas son excepciones a la regla. La determinación del ALBA-TCP para diversificar -en un sentido cuantitativo y cualitativo- la naturaleza de sus socios comerciales ha resultado en valiosas relaciones con países como India, China, Rusia e Irán, así como en un esfuerzo concertado para aumentar la cooperación entre América Latina y África (ALBA-TCP, 2009c).

El ALBA-TCP representa una novedad importante no sólo para la política en América Latina sino también internacionalmente. Es imprescindible, por lo tanto, entender su naturaleza: su ideología, sus instituciones y cómo ha implementado sus proyectos de desarrollo. Sin embargo, el estudio del ALBA-TCP está, con carácter general, subdesarrollado. La sección siguiente reseñará el contenido

4 Para una lista exhaustiva de los proyectos grannacionales véase ALBA-TCP, 2009a.

5 Evidentemente esta acción estuvo influida no sólo por la presión externa, sino también por la oposición doméstica al ALBA-TCP por partidos del centro y derecha y miembros de la comunidad empresarial (El Tiempo, 2010). El comercio continúa entre Honduras y los miembros del ALBA-TCP. 
de la literatura académica sobre el ALBA-TCP para determinar cuáles son las áreas que requieren más investigación.

\section{LA NATURALEZA DE LA LITERATURA SOBRE EL ALBA-TCP}

La literatura sobre el ALBA-TCP se caracteriza por dos rasgos principales: $a$ ) la escasez de artículos enfocados principalmente en el ALBA-TCP, y $b$ ) un enfoque metodológico descriptivo carente de verificación empírica. Aunque la organización se menciona con frecuencia en la literatura de estudios latinoamericanos, ciencias políticas y relaciones internacionales, el trabajo académico centrado principalmente en el ALBA-TCP no es común. Además, la naturaleza metodológica de este trabajo académico es descriptiva en casi su totalidad. Las fuentes de reconstrucción de la narrativa histórica y el análisis político se limitan predominantemente a los documentos oficiales del ALBA-TCP y fuentes secundarias. Salvo pocas excepciones, no se ha realizado ninguna investigación de campo. Tal observación expone la debilidad principal de esta literatura: las críticas no se basan principalmente en la investigación empírica, sino en el análisis de los documentos de la fuente primaria, por lo cual reflejan un cierto sesgo ideológico. Independientemente de la naturaleza crítica de muchos de los artículos analizados aquí, la mayoría ven el ALBA-TCP con buenos ojos, es decir, consideran el surgimiento de la organización como una novedad positiva, sin tener en cuenta sus defectos. La sección siguiente reseńará esta literatura; la gama de definiciones que los autores ofrecen y las críticas principales sobre el ALBA-TCP.

\section{HACIA UNA DEFINICIÓN DEL ALBA-TCP}

Dada la naturaleza descriptiva de la literatura sobre el ALBA-TCP, muchos de los artículos se enfocan en los aspectos históricos, ideológicos y políticos de la organización. La mayor parte de las definiciones del ALBA-TCP aquí mencionadas combinan estos aspectos. Los elementos comunes incluyen: la combinación de lo ideológico y lo histórico para producir una conciencia social basada en el mito unificador del legado de los "libertadores" latinoamericanos; el uso de lo histórico-ideológico para dar forma a una identidad política que se define en los términos de la contra-hegemonía; el ALCA como el principal catalizador del surgimiento de esta identidad contra-hegemónica; y la incapacidad de las categorías preexistentes de integración para describir el desarrollo del ALBA-TCP. 
Christopher David Absell

\section{El ALBA-TCP como la construcción de una conciencia revolucionaria latinoamericana}

La definición de la identidad del ALBA-TCP es un asunto complejo; la naturaleza polifacética de esta identidad puede ser difícil de articular sin ambigüedades, teniendo en cuenta los distintos significados intersubjetivos que pueden definir sus relaciones con otros actores, incluyendo sus propios miembros. A pesar de las dificultades inherentes a este proceso, algunos autores han intentado definir los aspectos ideacionales de la organización en términos normalmente históricoideológicos. Thomas Muhr sostuvo que es crucial para entender la naturaleza del ALBA-TCP “...construcciones ideacionales y normativas" en su proceso de desarrollo. Estas construcciones representan la conversión de lo histórico y lo ideológico en los pilares normativos de un proyecto regional, como observó Muhr: “...en el ALBA una ética contra-hegemónica de solidaridad y cooperación se fusiona con lo histórico-ideológico en la construcción de un 'complejo regional' y una 'comunidad regional'... y aportando las bases de un proyecto de gobernanza regional”. Las múltiples referencias a los libertadores latinoamericanos (Simón Bolívar, José Martí, Augusto Sandino) en la retórica del ALBA-TCP son un intento de “...construir una conciencia e identidad regional, popular-revolucionaria...” para “... contrarrestar la monopolización histórica de las relaciones externas ('quién es amigo o enemigo') por las élites nacionales de los países” (Muhr, 2010a: 46-47).

La importacia de la construcción de una conciencia regional para el desarrollo del ALBA-TCP también se exploró en el trabajo de Ken Cole. Como Muhr, Cole definió el ALBA-TCP como un "...proyecto contra-hegemónico..." que fue “... fundado sobre la complementariedad, la solidaridad, la cooperación, la dignidad humana, el respeto a la diversidad social -en vez de sobre la competencia, la dominación, la explotación, el poder corporativo y la conveniencia económica" (Cole, 2008). Cole sostuvo que este proyecto se caracterizó no sólo por el desarrollo institucional sino también por la "concientización política;" es decir, la "emergente conciencia social" de América Latina. Cole definió el ALBA-TCP de dos maneras: como el ALBA, un proceso de organización institucional regional; y como el alba, o el amanecer de una conciencia social que refleja la sociedad latinoamericana y rechaza la falsa promesa de la ideología neoliberal: "Mientras que el ALBA y otras iniciativas institucionales y regionales definen el regional 'nosotros' ['we'], alba es el proceso de un 'nosotros' ['us'] continental emergente: una conciencia social compartida y en evolución en la que ciudadanos individuales pueden seguir siendo ellos mismos mientras avanzan y se desarrollan conjuntamente" (Cole, 2010: 326). De esta manera el autor definió la naturaleza polifacética de la identidad del ALBA-TCP en los términos de dos elementos interrelacionados: como una política 
de contra-hegemonía que reflejó tanto las relaciones de confrontación (externas) como las relaciones cooperativas (internas), y un elemento histórico-ideológico que intentó dotar a los pueblos de América Latina de una conciencia social regional de la que el ALBA-TCP fue una expresión.

Esta tendencia a definir el ALBA-TCP en los términos de elementos políticos e histórico-ideológicos es común en la literatura. En uno de los primeros artículos sobre el desarrollo del ALBA-TCP, Nayllivis Nathaly Naím Soto definió la organización como "...una respuesta histórico-política..." a los intentos de los Estados Unidos de "redefinir" su posición hegemónica regional a través de la implementación del ALCA. El elemento histórico, observó Naím Soto, fue un intento de “...retomar la idea del libertador Simón Bolívar de crear una Confederación Americana con las repúblicas independizadas de España, para hacer frente a los bloques económicos asiático, europeo y norteamericano". El elemento político fue el uso activo de esta idea para "...minimizar, en lo posible, el poder político de Estados Unidos y debilitar su propuesta de integración económica hemisférica..." (Naím Soto, 2004: 57). Aunque este autor definió estos elementos en los términos de una dicotomía, éstos son inseparables; no es posible comprender la naturaleza histórica de la organización sin examinar antes su naturaleza política y viceversa.

\section{El ALBA-TCP como un proyecto contra-hegemónico}

Aunque en muchos casos el ALBA-TCP no se define explícitamente como "contrahegemónico", sí se lo describe frecuentemente en los términos de una estructura rival; es una "respuesta" o un "desafío" o la "contención" o "anti-tesis" de la hegemonía estadounidense (Ruiz et al., 2004; Kellogg, 2007; Serbin, 2007; Borbón, 2009). La definición contra-hegemónica del ALBA-TCP se articuló por Muhr en una serie de artículos en los que sostuvo que como un "proyecto de globalización contra-hegemónica”, el ALBA-TCP “...compite con la globalización capitalista mundial, regional y nacionalmente y en una gama de escalas subnacionales/locales" (Muhr, 2010a: 6). Según el autor, esta definición del ALBA-TCP depende de una comprensión neo-gramsciana de la globalización hegemónica en la que una “...congruencia histórica” de fuerzas materiales, ideologías, e instituciones bajo la globalización puede permitir hablar de un 'bloque histórico trasnacional' gramsciano". En este sentido, Muhr se refirió al papel de una "clase capitalista trasnacional" cuyo papel hegemónico en el sistema internacional se institucionalizó a través de una "estructura global de gobernanza ('disciplinaria') regulatoria" que se manifestó como una telaraña internacional de instituciones multilaterales, acuerdos 
y redes de políticas. Dado el dominio estructural e ideológico de la globalización capitalista, Muhr argumentó que es posible plantear que "La resistencia colectiva exitosa... tiene el potencial de transformar las relaciones sociales en la creación de una estructura rival o una configuración alternativa de fuerzas" (Muhr, 2010b: 32). El ALBA-TCP, como observó Muhr, es una estructura rival.

La definición de Muhr del papel contra-hegemónico del ALBA-TCP desarrolló una crítica interesante de la teoría anti y alter-globalización. Este autor señaló que el movimiento anti-globalización, poblado por una colección heterogénea de grupos de protesta y ejemplificado por las protestas contra la Organización Mundial de Comercio en Seattle en 1999, fue criticado por ser "negativo-oposicional, e incluso nacionalista", al no haber puesto propuestas alternativas concretas sobre la mesa. Por otro lado, el movimiento de alter-globalización, ejemplificado por el Foro Social Mundial, intentó redefinir la globalización distanciándose del consenso neoliberal hacia "...la noción de una 'globalización progresiva,' o 'globalización-desde-abajo', a través del empoderamiento y organización de la gente local para tomar el control de sus recursos y la construcción de movimientos trasnacionales sociales" (Muhr, 2010b: 36). Muhr argumentó que los dos enfoques pasaron por alto el papel del Estado en el proceso de desarrollo de los movimientos contra-hegemónicos: “...la ausencia de teorías adecuadas del Estado y el poder en la literatura respectiva ha llevado a una sobre-estimación del potencial transformador de los movimientos anticapitalistas sociales trasnacionales". El ALBA-TCP como “...una alianza entre el 'Estado-en-revolución' y la 'sociedad organizada' en resistencia al capitalismo global...” es un caso ejemplar de cómo el Estado puede ser un factor en el desarrollo de la globalización contra-hegemónica. En este sentido, tanto los actores estatales como los no-estatales colaboran “.... través de conjuntos de procesos políticos, económicos, culturales y sociales que se refuerzan y constituyen mutuamente" (Muhr, 2010b: 42) para construir lo que Muhr llamó una "clase trasnacional revolucionaria" en un emergente "régimen trasnacional de gobernanza revolucionaria". Esa alianza puede efectivamente contrarrestar las manifestaciones (tanto ideológicas como institucionales) de la globalización capitalista hegemónica "...simultáneamente desde las escalas inter-estatales, inter-gubernamentales y trasnacionales...” (Muhr, 2010b: 43).

\section{El ALBA-TCP como una nueva forma de integración}

En un estudio comparativo del ALCA y el ALBA-TCP, José Gerson Revanales Monsalve observó que ninguna de las propuestas se refirieron a la creación de instituciones supranacionales como las de la Unión Europea o la Comunidad 
Andina de Naciones (CAN) y que esta ambigüedad institucional obstaculizó el proceso de integración: “... aunque el propio presidente Chávez ha dicho que el ALBA es una nueva forma de integración, encontramos que como tal, presenta algunas carencias estructurales e institucionales que dificultan el desarrollo de sus objetivos". El autor sostuvo que en términos de categorías preexistentes de integración el ALBA-TCP se debería considerar más como "...un acuerdo de amistad, ayuda y cooperación...” que como un modelo de integración económica (Revanales Monsalve, 2007: 448). Esa definición reflejó no sólo la ambigüedad institucional del ALBA-TCP, sino también la naturaleza teóricamente restrictiva de las categorías preexistentes de integración económica. Revanales Monsalve argumentó que el error de este reduccionismo teórico fue que fracasó en reconocer el papel de los elementos sociales en el proceso de integración:

... muchos políticos caen en el error de pensar que puede hacerse la integración partiendo de alianzas políticas y algunos economistas piensan que pueden existir procesos de pura integración económica sin implicaciones políticas, sin aceptar que en buena parte la integración la hacen los pueblos, los ciudadanos, se va forjando día a día, sobre la base de intereses y de decisiones comunes... (Ibid.: 450).

Larry Catá Backer y Augusto Molino observaron que esta ambigüedad institucional del ALBA-TCP fue un intento deliberado de evitar la naturaleza supranacional de otros esquemas de integración con el fin de mantener los derechos soberanos del Estado. De esta manera, no se perdió autonomía y el Estado siguió siendo el actor clave: "La interacción supranacional debe ser controlada por el Estado, servir a los intereses del Estado, y enfocarse en la política pública" (Backer et al., 2009: 157). Esta idea constituye la base del concepto de la "Grannacional": la integración política y económica que evita la formación de instituciones supranacionales a favor del mantenimiento de la soberanía del Estado. Mohsen Al Attar y Rosalie Miller sostuvieron que esta subversión deliberada de los esquemas tradicionales de integración fue en parte el resultado del proceso de descolonización en la región. Debido a la larga lucha por la autonomía nacional y la auto-determinación, estos países se cuidaron de delegar la toma de decisiones a autoridades supranacionales, especialmente a las administradas por los poderes ex-coloniales. El ALBA-TCP, sin embargo, es una forma nueva de integración que "...encuentra un método de reafirmar la soberanía del Tercer Mundo sin el rechazo de la colaboración multilateral necesaria para mejorar el bienestar social colectivo" (Al Attar et al., 2010: 355). Los autores observaron que mientras que algunos regímenes supranacionales apoyan la integración a través de la competencia (siendo un ejemplo el modelo de 
reconocimiento mutuo de la Unión Europea), el ALBA-TCP “...promueve la armonía, reconstituyendo las relaciones Estado-a-Estado de la competencia y el interés propio hacia la complementariedad y la ventaja mutua" (Ibid.: 356). El surgimiento y desarrollo del ALBA-TCP, como José Briceño Ruiz y Rosalba Linares argumentaron, subraya la necesidad de "...repensar los acuerdos subregionales existentes...” como la CAN, el Mercado Común del Sur (MERCosur) y la Comunidad del Caribe (CARICOM), para redefinir el proceso de integración y “...superar los obstáculos y debilidades que, para algunos, representa el proyecto de negociación ALCA...” (Ruiz et al., 2004: 38-39).

\section{LA NATURALEZA DEL ENFOQUE CRÍTICO}

Para comprender la naturaleza de la literatura sobre el ALBA-TCP, es útil dividirla en cuatro categorías de análisis crítico: $a$ ) los trabajos que no presentan un análisis crítico, $b$ ) los trabajos que contienen un análisis poco crítico, $c$ ) los trabajos que presentan contenido crítico considerable, y $d$ ) los trabajos que presentan un soporte empírico para su contenido crítico. Como se puede ver en la Figura 1, la mayoría de la literatura sobre el ALBA-TCP se encuadra en las dos primeras categorías y, debido a la carencia de soporte empírico, se caracteriza principalmente por tener un sesgo ideológico derivado de su dependencia de las fuentes (primarias) oficiales.

Figura 1. Porcentaje de participación en las categorías de análisis crítico en la literatura sobre el ALBA-TCP

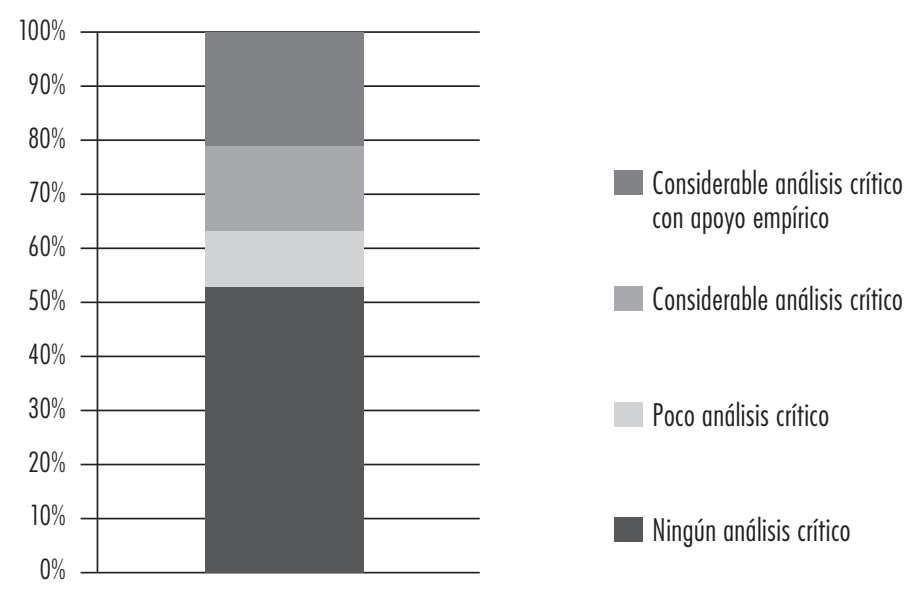


Los trabajos incluidos en la primera categoría (véase Cuadro 2) son en gran parte de carácter descriptivo y no presentan ningún análisis crítico del ALBATCP. Son principalmente discursivos, en su mayoría presentan el ALBA-TCP de una manera positiva (en algunos casos casi poética), y por lo general no proporcionan evidencia empírica para respaldar sus argumentos principales. Algunos (Naím Soto, 2004; Linares, 2007; Granato et al., 2010) son simplemente estudios históricos del desarrollo institucional del ALBA-TCP. Otros son decididamente más discursivos. La dependencia en la documentación oficial del ALBA-TCP como fuente primaria de información imbuye gran parte de este trabajo de un sesgo ideológico que no está respaldado por evidencia empírica. Un buen ejemplo del sesgo de selección (selection bias) ${ }^{6}$ que se puede derivar de este enfoque es evidente en el artículo de Eudis F. Fermín T. En este artículo Fermín analizó la naturaleza del ALBA-TCP durante el periodo 1999-2009 para determinar si “...representa realmente una ruptura paradigmática con los modelos económicos de los regímenes integracionistas imperantes en América Latina y El Caribe" (Fermín T., 2009: 342). Este enfoque metodológico, sin embargo, dependía de las declaraciones oficiales del ALBA-TCP que obviamente no proporcionaron una perspectiva ideológicamente neutral de la organización. Por esta razón, Fermín llegó a la conclusión de que el ALBA-TCP ciertamente representó una ruptura paradigmática con los regímenes integracionistas anteriores. El autor utilizó la opinión de la unidad de análisis para explicar su naturaleza.

Este enfoque es común en los otros artículos incluidos en esta categoría aunque -a diferencia de Fermín- la mayoría de ellos no definieron explícitamente su metodología cualitativa. Omaira García de Berrios sostuvo que el surgimiento del ALBA-TCP podría ser "el 'despertar de las Américas' para hacer realidad el sueño de desarrollo armónico de los pueblos" (García de Berrios, 2007: 629). Este argumento se derivó de un número limitado de fuentes (incluyendo Naím Soto; Ruiz et al., 2004) que proporcionaron un análisis poco crítico del ALBA-TCP. Otro ejemplo se puede encontrar en el trabajo de Cole. En un artículo elocuente que estableció un paralelismo con El amor en los tiempos de cólera de Gabriel García Márquez, Cole observó que "El poder trascendental del amor predominante sobre cólera (la globalización) está encapsulado en la iniciativa ALBA/alba como una estrategia de desarrollo endógeno regional, que es el proceso en el que el continental 'nosotros' permite a los ciudadanos individualmente

6 Para una explicación del sesgo de selección en el contexto de las ciencias sociales véase Thies, 2002: 355 . 
seguir siendo ellos mismos..." (Cole, 2010: 328; énfasis en el original). La prosa elocuente de Cole, sin embargo, no tuvo ningún apoyo empírico aparte de la referencia a los documentos oficiales y la retórica de las élites del ALBA-TCP (incluyendo Castro y Chávez).

Cabe destacar que muchos de estos trabajos no están completamente desprovistos de contenido crítico, aunque este contenido no se enfoca en el ALBA-TCP sino más bien en lo que se percibe como fuerzas externas negativas, tales como la arquitectura desigual del orden legal internacional (Al Attar et al., 2010), la naturaleza nefasta del neoliberalismo (García de Berrios, 2007; Kellogg, 2007), las tendencias destructivas de la globalización (Cole, 2010), o la falta de gobernanza regional en América Latina (Manzur et al., 2007). En general se presenta el ALBA-TCP como una fuerza positiva que contrarresta estas tendencias. En un estudio comparativo de la Unión de Naciones Suramericanas (UNASUR) y el ALBATCP, Paul Kellogg argumentó que si bien estas organizaciones desafiaron el papel de los Estados Unidos en América Latina, ambas fueron en esencia contradictorias en su naturaleza. Mientras que la UNASUR "... está completamente incrustada en una lógica muy familiar de acumulación de capital y poder corporativo", Kellogg observó que el ALBA-TCP “....está estrechamente asociado con los movimientos de masas...” (Kellogg, 2007: 189). La UNASUR es una manifestación de los gobiernos latinoamericanos "nuevamente seguros", reforzados por las exportaciones de energía y un consenso ideológico casi unánime, capaces de articular paradigmas alternativos de desarrollo que rechazan la hegemonía estadounidense. El ALBA-TCP, por otra parte, refleja la naturaleza de los movimientos sociales de América Latina, que se manifiesta a través de las “...ocupaciones de las fábricas en Argentina y Venezuela, las ocupaciones de tierra en Bolivia, Venezuela y Brasil, y la elección de gobiernos de izquierda a lo largo de la región" (Ibid.: 209). Kellogg observó que "... hay un sentimiento auténtico de que lo que estamos presenciando es... finalmente una alternativa a la larga noche del neoliberalismo" (Ibid.: 189). Sin embargo, como el trabajo de García de Berrios y Cole, este "sentimiento" y el discurso que lo acompaña se apoyó sólo en las declaraciones del ALBA-TCP y otras fuentes ideológicamente complementarias.

Otro ejemplo de un artículo generalmente crítico que fracasó en analizar críticamente el ALBA-TCP es el de Al Attar y Miller. Los autores presentaron una crítica pertinente del régimen legal internacional y una evaluación de los enfoques existentes procedentes del Tercer Mundo sobre el derecho internacional, y sostuvieron que el derecho internacional representa esencialmente una continuación de la época colonial: "Moldeado para proporcionar una justificación racional y moral de los horrores que visitaron a los pueblos y tierras re-descubiertos por las potencias europeas emergentes, el jus gentium se mantiene como el instrumento 
del colonialismo por excelencia" (Al Attar et al., 2010: 359). Aunque los enfoques procedentes del Tercer Mundo intentaron redefinir el derecho internacional en términos más pluralistas y democráticos, los autores observaron que el proyecto del Tercer Mundo se estancó por la falta de un "programa emancipador" viable y bien articulado. El ALBA-TCP, sin embargo, podría proporcionar un marco para la continuación del proyecto: “...potencialmente el ALBA podría llegar a las periferias del Tercer Mundo y, en el proceso, construir un etos de complementariedad que pueda informar a un nuevo marco legal internacional" (Ibid.: 355). No obstante, este argumento no se basó en la realidad empírica del ALBA-TCP, sino más bien en las declaraciones oficiales de la organización.

Cuadro 2. Los artículos de las categorías de análisis crítico

\begin{tabular}{|c|c|}
\hline Categoría de Análisis Crítico & Artículo \\
\hline Ningún análisis crítico del ALBA-TCP & $\begin{array}{l}\text { Al Attar et al., 2010; Cole, 2008, 2010; Fermín T., 2009; García de Berrios, } \\
\text { 2007; Granato et al., 2010; Kellogg, 2007; Linares, 2007; Morales Manzur } \\
\text { et al., 2007; Naím Soto, 2004. }\end{array}$ \\
\hline Poco análisis crítico del ALBA-TCP & Revanales Monsalve, 2007; Ruiz et al., 2004. \\
\hline Considerable análisis crítico del ALBA-TCP & Backer et al., 2009; Serbin, 2007; Siptroth, 2007. \\
\hline $\begin{array}{l}\text { Considerable análisis crítico del ALBA-TCP } \\
\text { con apoyo empirico }\end{array}$ & Borbón, 2009; Muhr, 2008, 2010a, 2010b. \\
\hline
\end{tabular}

A pesar de que los trabajos incluidos en la segunda categoría (Cuadro 2) presentan críticas al ALBA-TCP, estas críticas no se desarrollan (Ruiz et al., 2004) o se les resta importancia a favor de la organización (Revanales Monsalve, 2009). En uno de los primeros estudios comparativos del ALCA y el ALBA-TCP, Ruiz y Linares sostuvieron que éste tenía tres debilidades principales: $a$ ) un déficit democrático en su aparato organizacional; $b$ ) la naturaleza unilateral de su desarrollo, y c) la falta de claridad sobre la relación entre el ALBA-TCP y otras iniciativas regionales como la UNASUR (Ruiz et al., 2004: 41). Aunque ciertamente éstas fueron críticas pertinentes, sus autores no las desarrollaron en detalle, a diferencia de su crítica del ALCA que se basó en un considerable análisis macroeconómico. Como mencioné previamente, el artículo de Revanales Monsalve criticó al ALCA y al ALBA-TCP por su carencia de bases teóricas y supranacionales. A pesar de estas debilidades, el autor sostuvo que el ALBA-TCP "...pudiera ser un nuevo modelo de integración... fundamentado en los objetivos tradicionales como son el bien común y el desarrollo humano..." (Revanales Monsalve, 2009: 450). De esta manera, Revanales Monsalve descartó la importancia de las debilidades 
evidentes en la naturaleza institucional y organizacional del ALBA-TCP a favor de una perspectiva impregnada con el sesgo ideológico de las fuentes primarias de las que se derivó.

Los trabajos incluidos en la tercera categoría (Cuadro 2) presentan algunas críticas considerables al ALBA-TCP. Estas críticas no han sido verificadas empíricamente $y$, en este sentido, sólo representan una lectura crítica de las mismas fuentes primarias que los autores utilizaron en las dos primeras categorías. Andrés Serbin reflejó la primera crítica de Ruiz y Linares cuando argumentó que el ALBA-TCP sufrió de un "déficit democrático" en su aparato organizacional. En un estudio comparativo del ALCA, la UNASUR y el ALBA-TCP, el autor observó que “...es evidente que la emergencia del ALba con su énfasis social, y de las políticas regionales de Chávez... no dejan de introducir en la agenda de la integración regional la dimensión social y política frecuentemente obviada en el pasado" (Serbin, 2007: 199). Paradójicamente, Serbin observó que “...en términos de participación de la sociedad civil, el ALBA sólo ofrece, hasta ahora, la realización del Primer Encuentro de Movimientos Sociales por el ALBA en Venezuela y la potencial incorporación de un Consejo de Movimientos Sociales a su estructura formal..." Además, a pesar de “...la apertura a las propuestas desde los movimientos sociales..." el nexo del poder de la toma de decisiones se mantuvo en el Estado. Efectivamente, esta crítica subrayó una de las contradicciones más grandes del ALBA-TCP: la tensión entre la dependencia de los miembros en la explotación de los recursos naturales y la representación de una "sociedad organizada" opuesta en gran parte a esta explotación por razones sociales y ambientales. Utilizando el proyecto del ducto de gas natural que los gobiernos de Venezuela, Argentina y Brasil anunciaron en $2006^{7}$ como un ejemplo del déficit democrático, Serbin sostuvo que el ALBA-TCP “....ha tenido poca contemplación con los planteamientos críticos de algunos movimientos sociales o la participación de sus ciudadanos en los acuerdos interestatales firmados" (Serbin, 2007: 204).

Otro análisis crítico del ALBA-TCP fue realizado por Steven Siptroth. Después de una revisión de las propuestas del ALCA y el ALBA-TCP y una consideración sobre cómo podrían afectar la soberanía estatal, los derechos civiles y políticos, los derechos sociales y económicos, y la protección de los inversores, Siptroth (como Revanales Monsalve) concluyó que ambas eran "inadecuadas individualmente" como propuestas de integración: "El problema de ambas propuestas es que están diametralmente opuestas en el espectro socioeconómico. Debido a sus posiciones polarizadas la una contra la otra, ninguna, tal y como está diseñada

El Gran Gasoducto del Sur. 
actualmente, tiene el potencial para ganar la aceptación de todos los países de las Américas" (Siptroth, 2007: 391). El ALCA se presentó como un agente de la reforma neoliberal que "...tiene el objetivo de crear riqueza a través de la reducción de las barreras al comercio, el aumento de la eficiencia macroeconómica e institucional, y garantizar la protección del inversor y el acceso al mercado”. El ALBA-TCP, por otro lado, “...busca mejorar las condiciones sociales en los Estados miembros a través de la creación de un medio ambiente económico cooperativo que crea riqueza a través de un significativo control estatal de la gestión de los recursos naturales" (Siptroth, 2007: 390). Siptroth concibió una solución que se situó en un punto intermedio entre las dos propuestas: "Una alternativa mejor sería combinar los objetivos de bienestar social de la Alternativa Bolivariana para las Américas con el mercado abierto y las reducciones arancelarias prometidas por el Área de Libre Comercio de las Américas. La propuesta de un "Área de Libre Comercio de las Américas-Plus" prometería un medio efectivo de unificación económica para las Américas" (Siptroth, 2009: 361).

Backer y Molina presentaron un estudio exhaustivo de los elementos históricos, ideológicos e institucionales del ALBA-TCP y también intentaron contextualizar a la organización dentro del spaghetti bowl de acuerdos en América Latina. A diferencia de Serbín y Siptroth, el artículo de Backer y Molino no sacó una conclusión crítica (los autores reconocieron que tal conclusión sería prematura). En cambio, los autores destacaron algunos puntos de tensión que reflejaron algunos aspectos de los análisis críticos de los autores mencionados anteriormente. Estas tensiones incluyeron la carencia de instituciones supranacionales del ALBA-TCP, su dependencia en la integración manejada por el Estado, su naturaleza explícitamente ideológica (anti-capitalista y anti-estadounidense), su rechazo implícito de los modelos tradicionales de integración, y la ambigüedad de su posición hacia otros acuerdos (como la CARICOM) (Backer et al., 2009: 163-168).

Los trabajos de la cuarta categoría (Cuadro 2) presentan un análisis crítico considerable así como una verificación empírica para apoyar este análisis. Estos trabajos representan no sólo los estudios más desarrollados del ALBA-TCP, sino también los más precisos metodológicamente. Además ocupan una posición menor en la literatura; esta categoría únicamente engloba a dos autores: Muhr y Borbón. En una serie de artículos, Muhr desarrolló una sofisticada concepción teórica del ALBA-TCP como resultado de una investigación cualitativa considerable. La investigación de Muhr se enfocó en la naturaleza de los proyectos de desarrollo del ALBA-TCP en los campos de la educación, la salud y la energía en Nicaragua, y la "regionalización" del plan venezolano de la educación superior (Muhr, 2008; 2010a). Este trabajo de Muhr combinó la investigación etnográfica, las entrevistas a élites y el análisis del discurso, para proporcionar una perspectiva de la naturaleza ideológica y 
política del ALBA-TCP y de cómo ésta se ha traducido en proyectos concretos de desarrollo a nivel nacional y sub-nacional. Las conclusiones resultantes de esta investigación a veces contradijeron a autores cuyos argumentos se basaron en las fuentes primarias. El trabajo de Muhr, por ejemplo, se opuso al argumento de que el ALBA-TCP estaba dotada de un déficit democrático. El autor sostuvo que la naturaleza de los proyectos del ALBA-TCP en Nicaragua demostraron que "...la construcción del ALBA 'por los pueblos' y 'de abajo hacia arriba' no es mera retórica”. Utilizando ejemplos como el plan para el abastecimiento de petróleo ALBA Petróleos de Nicaragua (albanic), la campaña de alfabetización Yo Sí Puedo, y el programa oftalmológico Misión Milagro, Muhr observó que el ALBA-TCP construye una "sociedad regional" "...de abajo hacia arriba a través de la interacción trasnacional entre los Estados y los actores de la sociedad civil”. Estos tres ejemplos “... fueron directamente iniciados, negociados o coordinados desde el nivel de la sociedad civil y/o municipal” (Muhr, 2008: 159).

Otro artículo que utilizó una cantidad considerable de datos estadísticos para apoyar su análisis crítico fue el estudio de Josette Altmann Borbón sobre las políticas de la energía y el papel del PETROCARIBE en América Latina y el Caribe. El argumento de Borbón contradijo la perspectiva que establecía que el ALBA-TCP era un instrumento de coerción política venezolana. La autora sostuvo más bien que los países de América Central aprovecharon la iniciativa con sus propios intereses económicos en mente: “...su adhesión no implica necesariamente un compromiso ideológico-político, sino una voluntad de aprovechar las oportunidades económicas" (Borbón, 2009: 127). Borbón concluyó que esta tendencia explicó la membresía relativamente pequeña del ALBA-TCP en comparación con la de PETROCARIBE.

\section{CONCLUSIÓN}

En esencia, gran parte de la literatura revisada aquí observa el romance del ALBATCP pero no su realidad operativa. Esto no quiere decir que la mayoría de los artículos no sean críticos, sino que la suposición subyacente de casi todo el trabajo es que la organización es un factor positivo que podría llevar un elemento de equidad económica y justicia social a los pueblos de América Latina y El Caribe. Ciertamente, en el papel la naturaleza del ALBA-TCP y sus proyectos de desarro-

llo parecen ser una alternativa viable al neoliberalismo del ALCA y el éxito limitado de los otros proyectos de integración en la región. Sin embargo, la promesa del ALBA-TCP requiere más que una mera evaluación descriptiva. Su complejidad 
-en términos organizacionales e ideológicos- requiere de una investigación empírica exhaustiva llevada a cabo en el marco de una agenda bien definida.

La descripción histórica es un elemento necesario en el proceso de investigación. Esta reseńa muestra que los elementos descriptivos del desarrollo del ALBA-TCP hasta la fecha han sido explicados detalladamente. La ideología del ALBA-TCP ha sido examinada (más notablemente en el caso de Cole y Backer y Molina) de una manera creativa y sofisticada. Mientras que se necesita trabajar más en esta área, las bases se han sentado con éxito. El relato de la creación del ALBA-TCP, por lo tanto, ha pasado por su etapa de formación.

Las consecuencias de este desarrollo, sin embargo, no han sido estudiadas en gran detalle. Generalmente el estudio del ALBA-TCP carece de validación empírica. Hemos aprendido sobre la formación de las "empresas grannacionales", pero ¿sabemos algo sobre su aplicación? En concreto, ¿cómo han mejorado los proyectos de desarrollo del ALBA-TCP el bienestar de los pueblos de sus miembros? ¿Cómo difieren los proyectos entre los países? Aunque el ALBA-TCP sólo posea una vida organizacional de siete años, es evidente que muchos de sus proyectos han estado operando durante gran parte de este tiempo. Existe una oportunidad para examinar los resultados del desarrollo del ALBA-TCP.

Una de las debilidades principales de la literatura es la ausencia del desarrollo de una bibliografía compartida. Con pocas excepciones, muchos de los artículos no hacen remisiones. Por esta razón, frecuentemente adoptan argumentos alternativos ( $\mathrm{y}$ a menudo opuestos). Esta masa de argumentos en conflicto refleja la ausencia de un consenso para desarrollar una agenda de investigación sobre el ALBA-TCP. Espero que esta reseña de la literatura proporcione una plataforma temporal para esta agenda.

Y ahora, ¿̇en qué dirección seguimos? En el Cuadro 3 se presenta una lista de proyectos activos y propuestos que he adoptado de Muhr y expandido en algunas áreas, así como una lista del trabajo que se ha completado con éxito en ciertas áreas. Como se puede ver, el alcance de los proyectos del ALBA-TCP es bastante extenso, yendo desde las políticas monetarias y financieras hasta las iniciativas culturales. Sin embargo, también hay que señalar que la investigación realizada en estas categorías ha sido limitada. Las categorías presentadas aquí, como la seguridad alimentaria e industria y comercio, son áreas en las que los proyectos de investigación podrían enfocarse. Dada la naturaleza sin precedentes de algunos de estos proyectos, es necesaria más investigación no sólo para aumentar nuestro conocimiento sobre los proyectos de desarrollo del ALBA-TCP sino también para determinar si la naturaleza actual de estos proyectos confirma el consenso de la literatura. De tal manera, podríamos desechar esta mirada con buenos ojos a favor de una perspectiva más clara. 
Christopher David Absell

Cuadro 3. Áreas de investigación sobre los proyectos del ALBA-TCP (Muhr, 2010b; Portal ALBA-TCP)

\begin{tabular}{|c|c|c|}
\hline Categoría & Proyecto & Artículo \\
\hline $\begin{array}{l}\text { Seguridad } \\
\text { alimentaria }\end{array}$ & $\begin{array}{l}\text { "Misiones" alimentarias. Programa Ganadero del ALBA. PESCALBA. } \\
\text { GNC. ALBA-Alimentos. GNC-Soya. Casas de ALBA. }\end{array}$ & \\
\hline Cultura & GNP ALBA-Cultural. TELESUR. RADIOSUR. Librerías del Sur. & \\
\hline Energía & $\begin{array}{l}\text { PETROAMÉRICA (PETROANDINA, PETROSUR, PETROCARIBE). OPPEGASUR. } \\
\text { GNC-Petroalba, GNC-Petrosuramérica, GNC-Energía. }\end{array}$ & Borbón, 2009. \\
\hline Industria y comercio & $\begin{array}{l}\text { Factorías Recuperadas, Empresas Mixtas, GNE Aluminio, GNE Hierro } \\
\text { y Acero, GNE ALBA-Madera, GNE de Importaciones y Exportaciones } \\
\text { (ALBAEXIM). }\end{array}$ & \\
\hline $\begin{array}{l}\text { Educación y } \\
\text { conocimiento }\end{array}$ & $\begin{array}{l}\text { GNP ALBA-Educación. GNP Alfabetización y Post-Alfabetización. UNIALBA } \\
\text { (Universidad de los Pueblos de ALBA). UNISUR (Universidad de los } \\
\text { Pueblos del Sur). Escuela Latinoamericana de Medicina (ELAM). } \\
\text { Instituto Agro-Ecológico Latinoamericano "Paulo Freire” (IALA). ALBA } \\
\text { Energía Centro de Formación. }\end{array}$ & Muhr 2008, 2010a. \\
\hline $\begin{array}{l}\text { Protección } \\
\text { medioambiental y } \\
\text { cambio climático }\end{array}$ & $\begin{array}{l}\text { Misión Revolución Energía Internacional. ALBA GNPS } \\
\text { medioambientales. }\end{array}$ & \\
\hline Salud y medicina & GNP ALBA-Med. "Misiones" de salud. & \\
\hline $\begin{array}{l}\text { Monetaria/ } \\
\text { financiera }\end{array}$ & $\begin{array}{l}\text { GNP Comercio Justo. Sistema Unitario de Compensación Regional } \\
\text { (SUCRE). Banco del alba (BANCOALBA). Banco del Sur (BanCOSUR). } \\
\text { Fondo de Estabilización Macro-económico. Fondo de Reservas } \\
\text { Sudamericanas. }\end{array}$ & $\begin{array}{l}\text { Varios autores, 'South } \\
\text { America and the New } \\
\text { Financial Architecture,' } \\
\text { Journal of Post Keynesian } \\
\text { Economics, Vol. 32, No. } \\
\text { 2, invierno 2009-10; } \\
\text { Lourenç, 2010. }\end{array}$ \\
\hline Ejército & Comité Permanente de Defensa y Soberanía. & \\
\hline Ciencia y tecnología & ALBA-1 proyecto de cable de fibra-óptica. & \\
\hline Derecho & $\begin{array}{l}\text { El desarrollo de instituciones legales pluralistas y democráticas; } \\
\text { Convenciones constitucionales. }\end{array}$ & Al Attar et al., 2010. \\
\hline
\end{tabular}

\section{BIBLIOGRAFÍA}

ALBA-TCP, "Declaración conjunta entre el Presidente de la República Bolivariana de Venezuela y el Presidente del Consejo de Estado de la República de Cuba para la creación del ALBA", Portal ALBA-TCP, 2004a (consultado el 23 de mayo de 2010), disponible en http://www.alianzabolivariana.org/modules. php?name=Content\&pa=showpage\&pid=2060. 
ALBA-TCP, "Acuerdo entre el Presidente de la República Bolivariana de Venezuela y el Presidente del Consejo de Estado de Cuba, para la aplicación de la Alternativa Bolivariana para las Américas", Portal ALBA-TCP, $2004 \mathrm{~b}$ (consultado el 4 de octubre de 2010), disponible en http://www.alianzabolivariana. org $/$ modules.php?name $=$ News\&file $=$ article $\&$ sid $=81$.

"Declaración final de la primera reunión Cuba-Venezuela para la aplicación de la Alternativa Bolivariana para las Américas", Portal ALBA-TCP, 2005 (consultado el 9 de junio de 2010), disponible en http://www.alianzabolivariana.org $/$ modules.php?name $=$ Content $\& \mathrm{pa}=$ showpage $\&$ pid $=231$.

, "Comunicado conjunto", Portal ALBA-TCP, 2006 (consultado el 19 de junio de 2010), disponible en http://www.alianzabolivariana.org/modules.php?name=Content\&pa=showpage\&pid $=515$.

, "Conceptualización de Proyecto y Empresa Grannacional en el marco del ALBA", Portal ALBA-TCP, 2008 (consultado el 16 de junio de 2010), disponible en http://www.alianzabolivariana.org/modules.php?name=Cont ent\&pa=showpage\&pid $=2074$.

, “¿Qué es el ALBA-TCP?”, Portal ALBA-TCP, 2009a (consultado el 15

de abril de 2010), disponible en http://www.alianzabolivariana.org/modules.php?name=Content\&pa=showpage $\&$ pid $=2080$.

, "Declaración de la VI Cumbre Extraordinaria del ALBA-TCP", Portal ALBA-TCP, 2009b (consultado el 16 de junio de 2010), disponible en http:// www.alianzabolivariana.org $/$ modules. php?name=Content $\& \mathrm{pa}=$ showpage $\&$ pid $=1984$.

, "Declaración de Nueva Esparta II Cumbre América del Sur-África", Portal ALBA-TCP, 2009c (consultado el 4 de junio de 2010), disponible en http://www.alianzabolivariana.org/alba/archivos/declaracionfinalespanolfina.pdf.

, "Estructura y funcionamiento ALBA-TCP", ALBA-TCP.org, 2010 (consultado el 25 de mayo de 2010), disponible en http://www.alba-tcp.org/ content/estructura-y-funcionamiento-alba-tcp.

Al Attar, Mohsen and Rosalie Miller, "Towards an Emancipatory International Law: the Bolivarian Reconstruction", Third World Quarterly, vol. 31, num. 3, 2010, pp. 347-363.

Azicri, Max, "The Castro-Chávez Alliance", Latin American Perspectives, vol. 36, enero 2009, pp. 99-110.

Backer, Larry Catá and Augusto Molina, "Cuba and the Construction of Alternative Global Trade Systems: ALBA and Free Trade in the Americas", Pennsylvania Journal of International Law, vol. 31, num. 3, 2009, pp. 101-169. 
Borbón, Josette Altmann, "El ALBA, Petrocaribe y Centroamérica: ¿̨intereses comunes?”, Nueva Sociedad, vol. 219, enero-febrero 2009, pp. 127-144.

Cole, Ken, "ALBA: a Process of Concientizacion", The International Journal of Cuban Studies, vol. 1, num. 2, 2008 (consultado el 10 de enero de 2011), disponible en http://www.cubastudiesjournal.org/issue-2/international-relations/alba-a-process-of-concientizacion.cfm.

, "Jazz in the Time of Globalization: the Bolivarian Alliance for the Peoples of Our America”, Third World Quarterly, vol. 31, num. 2, 2010, pp. 315-332.

Comisión Económica para América Latina y El Caribe, Anuario estadístico de América Latina y El Caribe, United Nations Publications, Santiago, 2010.

"El Parlamento de Honduras ratifica su salida de la Alba", El Tiempo, 13 de enero 2010.

Fermín T., F. Eudis, "Alternativa Bolivariana para los Pueblos de Nuestra América: ¿̨la ruptura paradigmática de los modelos de integración?”, Espacio Abierto Cuaderno Venezolano de Sociología, vol. 18, núm. 2, 2009, pp. 339-365.

García de Berrios, Omaira, "Neoliberalismo: libertades desde los particulares para la negociación vs. ALBA: libertades desde el Estado para la integración”, Educere, vol. 11, núm. 39, 2007, pp. 629-637.

Granato, Leonardo and Nahuel Oddone, "Alianza Bolivariana y unASUR a la búsqueda de autonomía, orígenes y objetivos”, Intellector, vol. 7, núm. 13, 2010, pp. 1-25.

"Imposible ser parte de alianza que ataca a EUA", El Heraldo, 2 de febrero 2010.

Kellogg, Paul, "Regional Integration in Latin America: Dawn of an Alternative to Neoliberalism?”, New Political Science, vol. 29, num. 2, 2007, pp. 187-209.

Linares, Rosalba, "ALBA integración y desarrollo: de ideas a realidades", Geoenseñanza, vol. 12, núm. 1, 2007, pp. 31-40.

Lourenço, Fabiano Crespilho, "El silencio académico sobre el Banco del Sur", Problemas del Desarrollo, Revista Latinoamericana de Economía, vol. 41, núm. 160, 2010, pp. 135-154.

Morales Manzur, Juan Carlos and Lucrecia Morales García, "Origen y naturaleza de la Alternativa Bolivariana para las Américas”, Polis, vol. 3, núm. 1, 2007, pp. 55-85.

Muhr, Thomas, "Nicaragua Re-visited: From Neo-liberal 'Ungovernability' to the Bolivarian Alternative for the Peoples of Our America (ALBA)", Globalization, Societies and Education, vol. 6, num. 2, 2007, pp. 147-161. 
Muhr, Thomas, "Counter-hegemonic Regionalism and Higher Education for all: Venezuela and the ALBA", Globalization, Societies and Education, vol. 8, num. 1, 2010a, pp. 39-57.

"TINA go home! ALBA and Re-theorising Resistance to Global Capitalism", Cosmos and History, vol. 6, num. 2, 2010b, pp. 27-54.

Naím Soto, Nayllivis Nathaly, "Alternativa Bolivariana para la Américas: una propuesta histórica política al ALCA”, Geoenseñanza, vol. 9 núm. 1, 2004, pp. 57-73.

Revanales Monsalve, José Gerson, "Estructura morfológica del ALBA: ni el ALBA ni el ALCA son esquemas de integración", Anuario de Derecho Internacional, vol. 23, 2007, pp. 437-450.

Romero G., Antonio F., "La integración y cooperación en América Latina y El Caribe y la emergencia de nuevos espacios de integración: el ALBA-TCP", FLACSO, 2010 (consultado el 5 de julio de 2010), disponible en www.flacso. org/uploads/media/Ponencia_Antonio_Romero.pdf.

Ruiz Briceño, José y Rosalba Linares, "Más allá del Chavismo y la oposición: Venezuela en el proceso del ALCA y la propuesta ALBA", Geoenseñanza, vol. 9, núm. 1, 2004, pp. 19-47.

Serbin, Andrés, "Entre unASUr y ALba: ¿otra integración (ciudadana) es posible?”, En Mesa, Manuela (ed.) Pazy conflicto en el siglo XXI: tendencias globales. Anuario 2007-2008, Madrid, ceipaz/Icaria, 2007, pp. 183-207.

Siptroth, Stephen M., "Welcoming all to a Table of Plenty: the Free Trade Area and the Bolivarian Alternative as Competing Means of Economic Integration in the Americas", UCLA Journal of International Law \& Foreign Affairs, vol. 359, 2007, pp. 359-391.

Thies, Cameron G., "A Pragmatic Guide to Qualitative Historical Analysis in the Study of International Relations", International Studies Perspectives, vol. 3, 2002, pp. 351-372.

Varios autores, "South America and the New Financial Architecture", Journal of Post Keynesian Economics, vol. 32, num. 2, invierno 2009-10. 